\title{
Leadership de l'innovation: un moteur pour la créativité individuelle
}

\section{Résumé:}

Cette communication propose de démontrer que l'innovation qui englobe la créativité, la création de valeur ainsi que la capacité d'une entreprise à trouver des solutions nouvelles, requiert des leaders très orientés sur l'innovation et qui sont exigeants face à leurs subalternes et prêts à les soutenir. Pour parvenir à stimuler la créativité individuelle, ces leaders de l'innovation doivent accomplir une série d'actions qui sont liées aux aspects matériel et immatériel de l'environnement du travail tel que laisser une certaine autonomie au créatifs, reconnaitre les capacités créatives, apporter suffisamment de ressources pécuniaires, humaines, physique.

Mots clés : Innovation, leadership, leaders de l'innovation, créativité

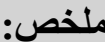

تقتر ح هذه الورقة البحثية تبيان أن الابتكار و الذي يعبر عن الإبداع و خلق القيمة إلى جانب قدرة المنظمة على تقديم حلول جديدة، يتطلب قيادة أكثر توجها نحو الابتكار، وأكثر إلحاحا تجاه الرؤوسين لتقديم الأفضل ومستعدة لتوفير كل أثنكال الدعم لهم. وبهدف تحفيز الإبداع الفردي، يتعين على قادة الابتكار في المؤسسة الالتزام بمجموعة من المهام و المسؤوليات كترك حرية أكبر للأفراد المبدعين، الاعتر اف بالقدرات الإبداعية، إلى جانب توفير ما يكفي من

$$
\text { الموارد المالية و البشرية والمادية . }
$$

الكلمات المفتاحية : الابتكار، القيادة، قيادة الابتكار ، الابداع

\author{
Korichi M ${ }^{\text {ed }}$ Salah \\ Labed Lazhar \\ Département de Sciences Economie \\ Université de Constantine 2
}

\section{Introduction :}

Depuis quelques années, les discours autour de l'innovation se sont multipliés, car elle doit permettre aux entreprises de perdurer et de se développer. Elle représente la clé de se différencier et de garder une avance face aux concurrents; elle est aussi le moyen de créer les marchés de demain ainsi que les emplois et les richesse futur des pays . 
L'innovation en tant qu'objectif, représente pour les dirigeant des entreprises un défi à relever, surtout par rapport aux moyens et aux conditions requises pour l'atteindre; en effet, les spécialistes s'accordent à reconnaître que la innovation au milieu du travail repose sur plusieurs conditions matérielles et immatérielles. Elle lui faut un environnement physique et culturel très ouvert, des équipes très motivées et créatives, Mais avant tout, l'innovation est une question de leadership.

Les leaders de l'innovation ont comme mission de bien mener leur équipes vers une production continue des idées innovantes, alors ils doivent créer l'environnement de travail adéquat et propice, constituer et stimuler leur équipes, les faire coexister et travailler ensemble même en acceptant les conflits d'idées. En outre, le rôle de vrai accompagnateur pour leurs subalternes, demeure une clé pour catalyser des efforts de création chez les individus créatifs.

Cette communication part de trois idées essentielles :

- La première est que l'innovation est désormais un choix incontournable pour la survie et la croissance de l'entreprise

- La deuxième est que le leadership est une nécessité pour mener efficacement le processus de l'innovation

- La troisième est que les leaders de l'innovation représentent un appui pour la créativité individuelle

Dans ce contexte, notre communication essayera d'apporter une réponse à la question suivante :

Quel sont les actions à mener par le leadership de l'innovation dans l'entreprise pour stimuler la créativité individuelle ?

Cette communication tire son importance du theme lui même, ce dernier qui suppose qu' un leadership qualifié est la reponse au problématique d'encadrement et de stimulation de l'innovation et la créativité au niveau des entreprise. Au fait, croitre la créativité individuelle en tant qu'objectif pour une entreprise exerçant dans un climat de concurrence ne peut se concrétiser sans un appui de leaders bien orienter sur l'innovation,

Afin de repondre à notre problematique, nous proposons de diviser notre communication en trois axes:

1- Leadership de l'innovation : cadres conceptuel

2- La créativité individuelle

3- Role du leadership de l'innovation dans la stimulation de la créativité individuelle

\section{1- Leadership de l'innovation : cadres conceptuel}

Après un court passage sur deux des principaux termes de notre recherche en l'occurrence l'innovation et le leadership, nous nous focalisons dans cette 
partie sur le Leadership de l'innovation comme nouveau concept dans l'ère du mangement .

\section{1-1- l'innovation}

En ce début de vingt et unième siècle, l'innovation est affichée dans les discours officiels comme une source de croissance et de progrès, le moyen privilégié à la fois d'un développement durable dans les pays dits développés et d'une amélioration du niveau de vie dans les pays en voie de développement.

\section{1-1-1-définition}

L'innovation est un terme polysémique que l'on emploie avec un sens différent selon le point de vue adopté. Nous retenons dans notre paper quelque définitions de ce terme.

D'abord, il est important de signaler que le terme innovation vient du latin Novus qui signifie nouveau. Le concept serait apparu en 1927 et désigne le fait d'introduire dans une chose établie quelque chose de nouveau [1] .

Certain auteurs considèrent l'innovation comme étant un processus permanent important pour la survie et le développement des entreprises, ces dernières doivent à présent pouvoir innover dans tous les aspects de leur organisation. [2]

L'OCDE définit l'innovation comme une mise en œuvre d'un produit (bien ou service) ou d'un procédé nouveau ou sensiblement amélioré, d'une nouvelle méthode de commercialisation ou d'une nouvelle méthode organisationnelle dans les pratiques de l'entreprise, l'organisation du lieu de travail ou les relations extérieures.[3]

D'un autre angle, l'innovation est considérée comme un processus concret qui se planifie, qui est réalisable et qui doit s'adapter aux besoins de l'entreprise. L'innovation est un moyen d'atteindre ses objectifs stratégiques, d'améliorer sa compétitivité, de se différencier et de créer de la valeur.[4]

Pour nous, l'innovation est l'ensemble d'activités aboutissants au lancement de nouveaux produits et/ou la mise en place de nouvelles méthodes et manières de gestion des pratiques de l'entreprise.

\section{1-1-2- Les enjeux}

L'innovation est plus que jamais investie de nouveaux rôles et fonctions. Elle serait une force motrice pour la survie des entreprises dans un environnement à forte concurrence, et c'est dans ce cadre que ces entreprises arrivent à réaliser les objectifs préétablis

Selon le rapport de 2001 du programme des nations unies pour le développement (PNUD),l'innovation est un moteur important dans l'amélioration des conditions de vie des pays pauvres[5], et les différentes innovations dans le domaine médical en sont un excellent exemple, ainsi l'innovation peut être un levier pour mettre des pays dans des trajectoires de développement et de prospérité. D'autre part, l'innovation est désigné comme 
étant à l'origine du renouvellement des stratégies industrielles et au développement de nouveaux secteurs d'activité économique.[6]

Une étude américaine citée par Loillier et Tellier en 99, a montré que les entreprises les plus performantes gêneraient prés de $50 \%$ de leur chiffre d'affaire avec des produits dans la durée de vie est de moins de cinq ans. Cette étude est étayée par les résultats de l'étude SESSI montrant que l'innovation est perçue par les industriels comme le moteur principal de leur développement.[7]

Tableau (1) Les motivations des entreprises pour innover

\begin{tabular}{|l|c|}
\hline \multicolumn{1}{|c|}{ Motivation pour innover } & \% d'entreprises \\
\hline Conquérir de nouveaux marchés & 57,8 \\
\hline Améliorer la qualité des produits & 50,2 \\
\hline Elargir la gamme des produits & 49,7 \\
\hline Remplacer des produits obsolètes & 23,6 \\
\hline Réduire les coutes salariaux par unité produite & 22,7 \\
\hline Satisfaire a une nouvelle norme & 22,5 \\
\hline Réduire ces consommations de matières & 17,6 \\
\hline Réduire les atteintes à l'environnement & 11,8 \\
\hline
\end{tabular}

\section{1-2-le leadership}

Source : SESSI, 1997

le leadership joue un rôle prépondérant dans la bonne gouvernance des affaires et pratiques des entreprise, et par conséquence dans la mise en exécution et la concrétisation des stratégies mises en place, notamment celle de l'innovation.

\section{1-2-1- definition}

Suivant leur spécialités et /ou la nature de leur fonctions, les chercheurs et praticiens ont tenter de définir le leadership.

L'approche axée sur les comportements qui se base sur le modèle de leadership charismatique, pense que le leader charismatique à une personnalité suffisamment forte pour exercer une influence significative sur ses subordonnés. le leadership charismatique à un esprit innovateur, une confiance en soi, une vision optimale du futur, une capacité de changement situationnel et finalement la morale et l'éthique. [8]

D'autre part, le leadership est perçu comme la manière d'influencer les personnes à se mobiliser pour concrétiser un objectif commun avec beaucoup de courage, de charisme et de talents.[9]

tandis que l'approche axée sur les situations, voit que le leadership le plus efficace dépend de la situation et des individus en place; Donc, le leader élabore un environnement susceptible afin de faciliter le rendement et de satisfaire le groupe.[10] 
Pour nous, Le leadership constitue la manière par laquelle une personne exerce une influence sur d'autres afin de réaliser un objectif commun en leur donnant une bonne vision de l'avenir pour avoir une action efficace.

\section{1-2-2-Les styles de leadership}

Les trois styles de leadership : le leadership transactionnel et le leadership transformationnel ont été mises en place par James Mc Gregor Burns.[11]

\section{-Le leadership transactionnel}

L'approche transactionnelle s'est basée sur les échanges de transaction qui s'entremêlent entre le leader et le subordonné sur le principe " donnant/donnant». Un leader transactionnel fournis de l'assistance aux autres en échange de leurs efforts afin d'améliorer le niveau ainsi que la qualité de la performance de ses subordonnés. Cela signifie que le leader donne une récompense en retour d'un comportement approprié

\section{-Le leadership transformationnel}

L'approche transformationnelle s'est basée sur les échanges de transformation entre le leader et le subordonné .Un leadership transformationnel est un processus qui provoque un engagement mutuel du leader et du subordonné placé sous sa dépendance hiérarchique basé sur l'adhésion commune. Aussi, ce type de leader est attentif aux intérêts des suiveurs. Pour cela, il augmente chez les subordonnés le désir de réussir en leur stimulant au travail sous de nouvelles perspectives avec 'un niveau de motivation et de satisfaction élevé.[12]

\section{-Le leadership « laisser faire »}

Le leader de type "laisser-faire » considère que son rôle prépondérant est de faciliter la tâche de ses subordonnés en leur fournissant l'information et en agissant comme lien entre le groupe et l'environnement extérieur[13] .Pour cela il utilise peu ou pas son pouvoir et laisse une liberte totale à ses subordonnés de choisir et d'atteindre les buts. ce type de leader comme un " non leader " puisqu'il n'a aucun rôle sur le comportement de ses subordonnés.

\section{1-3-Leadership de l'innovation}

Le besoin d'innovation pour les entreprises a donné lieu à une nouvelle vision sur le rôle des leaders dans la réussite des efforts de création, car Sans leadership de l'innovation, les entreprises perdent beaucoup face à la concurrence. Plusieurs chercheures ont définit le concept de leadership de l'innovation suivant leurs champs d'activités et leur spécialités.

Dans sa thèse de doctorat, Gliddon David Gregory, a définit le leadership de l' innovation comme étant une philosophie et technique qui combine différents styles de leadership pour influencer les employés pour produire des idées créatives, des produits et des services. [14] 
Et lors d'un interview accorder à innovation leadership forum, Betinna Von Stamm a définit le leadership de l' innovation comme la création d' un environnement où les travailleurs peuvent d'abord et avant tout être euxmêmes; et S'ils sont autorisées à être eux-mêmes, ils se sentent libres de s'exprimer, être créatif et de contribuer à l'innovation[15]

Pour notre part, le leadership de l'innovation est une manière de manager ses subalternes on les stimulant, les orientant et leur créant un climat de travail de qualité afin de les inciter à présenter des idées innovatrices .

Ces définitions nous laisse dire qu'un leader de l'innovation n'est pas forcement un génie créatif, c'est avant tout celui qui sait créer un climat qui permettra à son équipe d'oser proposer des idées nouvelle.

1-4-leaders de l'innovation et innovateurs?

il faut faire une distinction entre leaders de l'innovation et innovateurs; ce ne sont pas forcément les mêmes personnes.

Tableau( 2 ): leaders de l'innovation et innovateurs

\begin{tabular}{|c|c|}
\hline Un innovateur & Un leadership de l'innovation \\
\hline $\begin{array}{l}\text {-propose de idées innovantes, crée et } \\
\text { trouve des solutions nouvelles, } \\
\text { Introduit quelque chose de nouveau } \\
\text { (produit un procede, une method ....) }\end{array}$ & $\begin{array}{l}\text {-crée un climat propice pour innover } \\
\text {,motive, oriente ses subalternes } \\
\text { innovants, participe et propose des } \\
\text { idées }\end{array}$ \\
\hline
\end{tabular}

Source : les chercheurs

En effet, un leader de l'innovation ne doit pas nécessairement être celui qui propose des idées innovantes, mais il est celui qui appuyé son staff et le pousse à avancer leurs idées. Les entreprises peuvent avoir des innovateurs au sommet, mais il est plus important encore pour elles d'avoir des leaders de l'innovation. Steve Jobs était les deux à la fois: un formidable innovateur et un véritable leader de l'innovation.

\section{1-5 - A quel niveau peut-on trouver les leaders innovateurs ?}

Des études de cas concrets d'entreprises révèle une constante: les entreprises innovantes, quel que soit leur domaine d'activité, ont beaucoup de leaders de l'innovation, et ceux-ci se retrouvent à tous les niveaux .Au fait, ces entreprises innovantes ont en général des leaders de l'innovation à différents niveaux hiérarchiques dans leur organisation; dans les petites entreprises, on retrouve en général ces leaders de l'innovation au sommet de la hiérarchie. Dans les plus grandes entreprises, ils peuvent occuper des postes élevés, notamment au comité de direction, comme responsables d'entités opérationnelles ou de fonctions importantes. [16]

En outre, les leaders de l'innovation peuvent exercer à un niveau intermédiaire où ils jouent un rôle de transmission entre le sommet, les opérations et la base. En général, les leaders de l'innovation appartiennent à trois catégories distinctes de «champions». On trouve très souvent: [17] 
- des champions techniques dans les bureaux d'études ou les départements de $\mathrm{R} \& \mathrm{D}$; ce sont eux qui proposent des technologies ou des concepts de produits nouveaux.

- des champions commerciaux, capables de transformer les nouveaux produits ou services en flux de revenus.

- les champions exécutifs, ces leaders de haut niveau capables de prendre des risques, même par rapport à leurs collègues de direction, pour réaliser ces innovations.

2-La créativité individuelle

Selon le dictionnaire des concepts, la créativité est définie comme la capacité à imaginer rapidement différentes solutions originales, si l'on est confronté à une situation de problème[18]

Tandis qu' un individu créatif est une personne à concevoir une discussion conceptuelle puis à l'exploiter avec des idées existantes et / ou à l'explorer en imaginant des idées nouvelles[19]

La littérature spécialisé nous enseigne que la créativité est le résultat d'un processus de quatre étapes, que nous essayons de présenter.

\section{2-1-le processus créatif}

les étapes du processus créatif de la figure suivante sont génériques : des allers-retours sont nécessaires au cours du processus.[20]

Figure ( 1 ) : Aperçu de la créativité individuelle

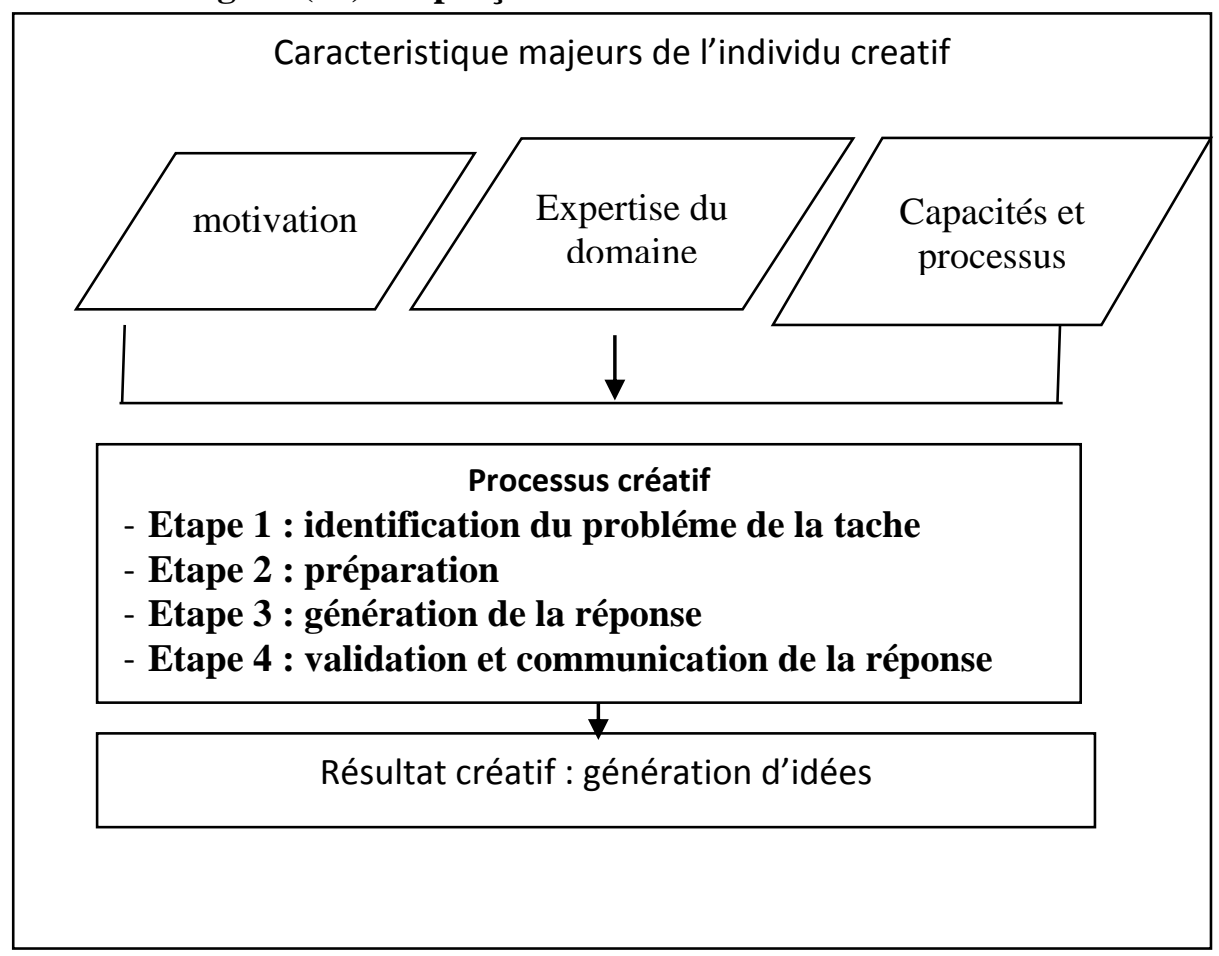


Source: ibid

- la première étape consiste à prendre conscience de l'existence d'un problème . l'individu peut s'en rendre compte par lui-meme; il peut recevoir les doléances d'une tierce perdonne ( un collégue, un client, un fournisseur, etc), ou encore son responsable l'affecte à un problème identifié par la hiérarchie.

-la deuxième étape : l'individu regroupe les informations disponibles, les analyse, les « triture » selon des méthodes créatives pour trouver une idée

-la troisième étape :étape de génération de la réponse

-la quatrième étape :l'individu teste son idée avant de la communiquer et qu'elle soit reconnue comme «LA » bonne solution au problème .

2-les sources de la créativité individuelle[21]

Des différentes études nous montrent que certaines qualité des individus créatifs sont à priori innées ou acquises par l'éducation, tandis que d'autre peuvent être influencées par des facteurs extérieures comme le management de l'innovation ou encore la culture de l'organisation.

-une relative autonomie

L'autonomie est un critère majeur dans la démarche de la créativité. Elle implique la capacité de l'individu à se créer ses propres lois. Par exemple, la créativité individuelle appréhendée sous le mode de l'exploration demande de concevoir par soi - même un modèle de raisonnement. Toutefois, cette autonomie est «relative»; elle ne peut se faire qu'à partir de l'individu, elle doit prendre en considération son contexte.

-les capacités spéciales pour penser de manière créative.

Une pensée divergente, une définition hors norme d'une situation ou encore une hétérogénéité des liens entre domaines sont quelques-unes des démarches de ces créatifs. des outils permettent, certes, de développer ces capacités, mais ils ont leurs limites.

\section{-Capacités sociales}

Une dernière capacité à noter place l'individu face à son entourage . ses capacités sociales le conduisent à ouvrir son esprit aux idées des autres, à les écouter et à développer et à maintenir un bon relationnel.

\section{-La motivation}

Les caractéristiques mentionnées précédemment dépendent pour partie d'un facteur majeur : la motivation . par définition, elle implique des forces consciente ou non qui déterminent le comportement d'une personne. Dans le cas de la créativité individuelle, les auteurs en psychologie en retiennent deux types : la motivation dite intrinsèque car provenant de l'individu, et la motivation extrinsèque car induite par des facteurs extérieurs à l'individu. 
Les caractéristique déjà cités sont un échantillon de traits d'un individu créatif . le tableau ( 3 ) donne plus de traits du créatif.

Tableau ( 3 ): caractéristique d'un individu créatif

Etre endurant, curieux, enthousiaste Disposer d'une belle énergie et d'une honnêteté Intelectuelle

Etre capable d'une relative autonomie Avoir le gout du défi
Détenir des capacités pour penser de manière créative, Etre attiré par le risque

Maintenir et développer une expertise Posséder des aptitudes sociales (etre ouvert, à l'écoute...)

Source : ibid

3-Leader de l'innovation : que pourrez - vous faire pour inciter la créativité individuelle?

Tout le monde s'accorde à reconnaître que l'innovation des ressources humaines repose sur plusieurs conditions matérielles et immatérielles . Elle lui faut un environnement physique et culturel très ouvert, des équipes très motivées et créatives, Mais avant tout, la créativité est une question de leadership.

Nous présentent dans cette dernière partie de recherché les Caractéristiques du leadership de l'innovation, et les actions à mener par ce leader pour stimuler la creativite individuelle

\section{3-1-Caractéristiques du leadership de l'innovation}

des chercheurs ont tenté de tracer un profil des leaders de l'innovation, ils croit que ces leaders partagent au moins six ou sept grands traits de comportement.

\section{- La créativité}

La créativité est devenue la compétence de leadership indispensable à n'importe quelle organisation. Ce résultat apparait d'après une étude d' IBM Institute for Business Value et d' IBM Strategy \& Change obtenue à partir de plus de $1500 \mathrm{CEO}$, directeurs généraux et hauts responsables des secteurs public et privé dans 60 pays et 33 secteurs d'activité. [22]

Les résultats montre que la «créativité » est la compétence de leadership primordiale pour les organisations de toute nature. Le climat d'incertitude serait mieux gérée par les dirigeants créatifs et ces derniers utiliseraient plus souvent que d'autres de nouveaux modèles d'affaires

\section{- Un mélange «d'émotion et de réalisme»} selon la formule proposée par Daniel Borel, le co-fondateur de Logitech, Cela veut dire une combinaison inhabituelle d'intérêt pour la créativité ainsi que pour la discipline nécessaire à l'exécution des projets. Certaines entreprises, comme Logitech, essaient de promouvoir 
des leaders qui cumulent ces deux intérêts, mais ce n'est pas le cas de toutes les entreprises[23]. Il est parfois plus facile, comme on le verra plus loin, de sélectionner certains leaders orientés vers la créativité et d'autres plus focalisés sur la discipline liée à l'exécution.

\section{- L'acceptation des risques et des échecs}

La deuxième caractéristique des leaders de l'innovation, est l'acceptation des risques et des échecs. Mais au delà, leur qualité première est sans doute leur aptitude à faire en sorte que leurs équipes apprennent le plus possible de leurs échecs. L'échec fait partie du jeu de l'innovation, même si des outils d'analyse peuvent le prévenir. Un bon leader doit savoir tirer les leçons d'un échec et, le moment venu, apprendre à son équipe à rebondir, sans se démoraliser.

\section{- Poser les bonnes questions}

les qualité des questions posées par le leader offrent aux subalternes la possibilité de produire de nouvelles idées, voir instaurer un climat créatif. Par exemple des questions comme: Lorsque nous avons innové avec succès, qu'est-ce que le nouveau produit ressemble? Quels problèmes résout-elle pour nos clients? Comment est-il apporte une nouvelle valeur sur le marché? peuvent inciter les travailleurs à produire de nouvelles idées. [24]

\section{- la capacité à attirer des innovateurs}

mais aussi à former et mener des équipes à compétences mutiples, et ce jusqu'à la réussite. Les équipes d'innovation qui réussissent rassemblent en général des individus à fort caractère - l'innovation ne naît pas à partir de consensus mous. Les leaders doivent savoir constituer ces équipes, les faire coexister et travailler ensemble en acceptant les conflits d'idées (et non de personnes) car seule la confrontation permet d'aller de l'avant et trouver des solutions innovantes. [25]

\section{- l'ouverture aux idées}

La cinquième qualité des leaders de l'innovation, c'est l'ouverture aux idées et technologies de l'extérieur et la capacité de convaincre leurs équipes d'aller chercher ces idées à l'extérieur au lieu d'essayer de réinventer la roue en interne. Cette approche, connue sous le nom d'Open Innovation et promue par des entreprises comme Procter \& Gamble, nécessite de favoriser la multiplication des réseaux externes permettant de mettre en contact l'entreprise avec des personnes ou des organisations externes innovantes. Certaines entreprises constituent ainsi leurs propres réseaux.

- la passion, pour leur mission et pour l'innovation

Tous font preuve d'ardeur pour motiver leurs équipes et leur communiquer leur enthousiasme. Lorsqu'il dirigeait Logitech, Daniel 
Borel aimait à dire: «On gagne notre vie, on existe, on survit par l'innovation. Si quelqu'un ne partage pas cette passion à chaque niveau, il ne pourra pas rejoindre Logitech». Appliquant cette idée à la lettre, Logitech s'assure que ses nouvelles recrues partagent tous cette passion lors de ses tests de sélection. [26]

\section{3-2-Action pour stimuler la creativite individuelle}

Teresa Amabile s'est intéressée au cadre de vie au travail comme une ressource à la créativité individuelle : que peut faire ( ou non) un leader pour inciter les personnes à libérer leur potentiel créatif ?

Dans leur livre $<<$ management de l'innovation $>>$, Sevrine le Loarne et Sylvie Blanco se sont intéressés au aspects matériel et immatériel du cadre de vie au travail comme étant un support pour booster la créativité individuel. Ils ont déterminé les actions les plus importantes d'un leader de l'innovation , qui sont : [27]

\section{-Laisser au créatif une autonomie ...relative}

L'individu créatif a besoin de bénéficier d'une relative autonomie. Le leader doit donc lui transmettre le sentiment qu'il contrôle lui-même son propre travail et ses idées, et qu'il est opérationnellement autonome. Cette liberté ne signifie pas que le manager ne contrôle pas du tout le projet du créatif. Au contraire, il doit s'intéresser au projet et poser des questions à la personne : " où en êtes-vous dans l'avancée du projet? quelles sont vos dernières idées ? souhaitez-vous communiquer cette pensée aux membres de l'équipe ? ». l'intérêt de poser des questions au créatif est de le rendre responsable de son projet. le leader ne doit donc pas hésiter à s'entretenir régulièrement (de manière formelle ou non) avec lui pour lui indiquer que son projet est important pour la dynamique d'innovation en place dans l'entreprise .

\section{-Reconnaissance}

Le leader doit identifier des stratégies de reconnaissance adaptées pour valoriser la réussite et le travail effectué. ainsi cette reconnaissance n'est pas axée uniquement sur le résultat ( approche téléologique) mais aussi sur le processus permettant ce résultat . les sont donc reconnues comme capitales- même en cas d'échec. De plus, ce système d'évaluation incite le créatif à ne pas se contenter de projets de court terme qui pourraient aboutir à des résultats plus certains.

\section{-Protéger le créatif des distractions}

si d'autres personnes de l'entreprise le sollicitent pour les aider dans une autre tâche, l'individu créatif peut perdre capacités créatives de vus son projet principale et ainsi se détourner subrepticement de ses objectifs. Le manager se doit d'assurer le confort intellectuel du créatif en lui évitant d'être sollicité pour des raisons externes au projet. En revanche, le 
créatif ne doit pas être déconnecté de son environnement car il en a besoin pour étalonner ses idées et trouver de nouvelles sources d'inspiration, notamment s'il cherche à fertiliser son champ d'étude par un autre domaine. Le leaderr et le créatif sont donc amenés à collaborer sur l'élaboration du cadre de travail -cadre qui évoluera certainement au fil de l'avancement du projet.[28]

\section{-Apporter suffisamment de ressources}

Si les distractions au niveau des idées peuvent éloigner le créatif de ses intentions mais aussi le stimuler, les difficultés matérielles peuvent rapidement nuire à la créativité individuelle. Dans l'idéal, le leader doit apporter suffisamment de ressources pécuniaires, humaines, physique pour décharger le créatif de ses demandes. Comment pourrait-il se concentrer sur les idées s'il est obligé de remplir des dossiers de demandes de matières premières nécessaires à la réalisation d'un prototype, pour ensuite s'enquérir de la suite de sa demande et attendre de les recevoir?.

\section{-trouver un équilibre entre « laisser du temps» et « être dans l'urgence»}

Teresa Amabile rappelle que le manager doit . le temps est nécessaire pour explorer , réfléchir, discuter, formaliser des modèles, etc . mais a pression du temps conduit un créatif à penser de manière pragmatique : " comment traduire mes cogitations en idées propices à l'innovation ? » autant l'absence de contrainte qu'une pression trop forte freinent la créativité individuelle dans une entreprise . les chercheurs montrent que la relation entre le « temps» et la « créativité individuelle » est curviligne. Le manager doit alors gérer le facteur « temps » au fil du projet . si , lors du lancement, le créatif a besoin de temps pour explorer des horizons variés, le manager rassurera cette liberté pour l'inciter à penser à la traduction de ces idées .

\section{-la capacité des personnes à collaborer entre elles}

$\mathrm{Si}$ les individus sont sur la défensive, ils ne partageront pas leurs discussion conceptuelles, ils manqueront d'honnêteté intellectuelle par peur de voir un autre s'approprier leurs idées. Le leader joue donc un rôle clé : il permet la communication entre les personnes de l'entreprise et s'assure du respect de la paternité des idées. Il peut ainsi intégrer ce comportement comme un critère d'évaluation du travail du créatif. Si un créatif s'estime dépossédé de son exploration et, par la suite, de ses idées, il participera difficilement à des projet collectifs supposant le partage de ses connaissances. Pour éviter cette situation dommageable à l'innovation, le manager s'assurera de la probité des acteurs conviés au partage et devra sanctionner un éventuel opportuniste. Ces signaux forts participeront à la création d'une véritable aventure créative entre les acteurs mais aussi au maintien de la créativité de l'individu . 


\section{conclusion}

l'innovation qu'est l'ensemble d'activités aboutissants au lancement de nouveaux produits et/ou la mise en place de nouvelles méthodes représente un besoin et est désormais un choix incontournable pour la survie et la croissance de l'entreprise. Ce besoin d'innovation pour les entreprises à donné lieu à une nouvelle approche sur l'importance qu'occupe les leaders dans la réussite des efforts de création, surtout si ces leaders arrivent à exercer efficacement leur influence sur leur équipes afin de les pousser à produire des idées créatives, des produits et des services. Au fait, les leaders d'innovation représentent un vrai appui pour la créativité individuelle dans l'entreprise, surtout lorceque ils mènent parfaitement leur actions requisent en l'occurrence la reconnaissance du travail effectué , l'assurance du confort intellectuel du creative.

\section{references}

1-Durieux F., «Management de l'innovation», éd. Vuibert, Coll. FNEGE, Paris, 2000

2-Qu'est-ce que L'innovation ? consulter dans (

https://www.economie.gouv.qc.ca)

3-OCDE: Manuel d'Oslo, principes directeurs pour le recueil et l'interprétation

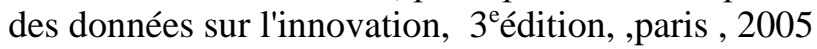

4- https://www.mapaq.gouv.qc.ca

5-Fernez -Walsh S., Romon F., «Dictionnaire du management de l'innovation», éd. Vuibert, Paris, 2008

6-Commission européenne, Livre vert sur l'innovation, décembre 1995

7-Amdaoud Mounir, La gestion de l'innovation dans les entreprises algériennes:enjeu majeur pour obtention d'un avantage concurrentiel durable,mémoire de magister en sciences de gestion, universite de tizi ouzou, 2014

8-Bereffas imene, et benabou diilali, Les pratiques de leadership dans les administrations publiques cas de quelques superviseurs Algériens, seminaire sur la grh dans les stuctures publiques, biskra , 2015

9- M. Bass Bernard \& Bruce J. Avolio.,"The Multi factor Leadership Questionnaire", 2000, in « comme exigence partielle de la maîtrise en gestion de projet », Suzanne Vincet, université de Québec, 2006.

10- Koontz \& O’Donnell., " Management Principes et méthodes de gestion, », In «types de manager et pratiques entrepreneuriales en Algérie (secteur public) », El Ghazi H., 2011

11-S.M Sarah., \& Nouchka W., « Style de leadership, LMX et engagement organisationnel des salariés : le genre du leader a-t-il un impact? », Laboratoire Prism Cergors, Université Paris 1 Panthéon Sorbonne,2009

12-Op.cit, Suzanne V., 2006.

13- Op cit , Bereffas imene , et benabou diilali , 2015 
14-G , Gliddon. David,. Forecasting a competency model for innovation leaders using a modified Delphi technique,Doctoral dissertation in philosophy , Pennsylvania State university , 2006

15- Betinna von stamm, innovative leadership what is it? Consulter dans (innovationleadershipforum.org/)

16-Jean-Philippe Deschamps, 1 'innovation et le leadership, revue économique et sociale, n 4 December 2012

17-Ibid

18-RAYNAL F. et RIEUNIER A., Pédagogie : Dictionnaire des concepts clés, 5ème édition, , Édition ESF, Paris Éditeur2005

19-Le Loarne, S et Blanco S, Management de l'innovation, Pearson

éducation , France, 2011

20-Ambile, $\mathrm{T}$ et al, time pressure and creativityin organizations : a

longitudinal field study, HBS working paper, 2002

21- Op cit, Le Loarne, $\mathrm{S}$ et Blanco S, 2011

22- Gaffard Jean-Luc et Zieger Jean , Economie et management de la créativité : les Journées de l'Université Sophia Antipolis , , 18-20 octobre, 2012

23-Op cit, Jean-Philippe Deschamps 2012

24-http://www.forbes.com

25-Op cit , Jean-Philippe Deschamps, 2012

26- ibid

27- Op cit, Le Loarne, S et Blanco S, 2011

28-ibid 